Daimon. Revista Internacional de Filosofía, $n^{\circ} 84,2021$ pp. 5-9

ISSN: 1130-0507 (papel) y 1989-4651 (electrónico)

http://dx.doi.org/10.6018/daimon.485241

Licencia Creative Commons Reconocimiento-NoComercial-SinObraDerivada 3.0 España (texto legal). Se pueden copiar, usar, difundir, transmitir y exponer públicamente, siempre que: i) se cite la autoría y la fuente original de su publicación (revista, editorial y URL de la obra); ii) no se usen para fines comerciales; iii) se mencione la existencia y especificaciones de esta licencia de uso.

(c) (1) (3)

\title{
Presentación del número monográfico sobre Expressing Hatred: The Political Dimension of Expressives
}

\author{
Presentation of the monographic issue on \\ Expressing Hatred: The Political Dimension of Expressives
}

\author{
EDUARDO PÉREZ-NAVARRO* \\ MARÍA J. FRÁPOLLI**
}

\begin{abstract}
Resumen: Desde hace unos años, se ha producido dentro de la filosofía analítica un movimiento de acercamiento a las prácticas reales y de huida de las idealizaciones no justificadas que pretende poner las herramientas conceptuales desarrolladas durante el último siglo al servicio de la justicia social. En el ámbito de la filosofía del lenguaje, este giro ha pasado por el análisis de expresiones del lenguaje natural que, por no encajar de forma completamente satisfactoria con la concepción del significado como condiciones de verdad, han recibido tradicionalmente poca atención. Sin embargo, estas expresiones juegan un papel fundamental en la comunicación con impacto político. Hablamos de los expresivos, esto es, expresiones que se utilizan para comunicar una cierta actitud. El propósito de este número especial de Daimon es ofrecer una panorámica de algunos debates que se están desarrollando en la actualidad en relación con la dimensión política de los expresivos, pero también de discusiones cercanas que en ocasiones se solapan con esta, tanto en filosofía del lenguaje como en ramas de la filosofía afines.
\end{abstract}

Palabras clave: expresivos, filosofía del lenguaje, giro político de la filosofía analítica, injusticia

\begin{abstract}
For some years now, there has been a movement within analytic philosophy to get closer to real practices and to flee from unwarranted idealizations in order to put the conceptual tools developed over the last century at the service of social justice. In the field of philosophy of language, this turn has involved the analysis of natural language expressions that, not fitting in a completely satisfactory way with the conception of meaning as truth-conditions, have traditionally received little attention. However, these expressions play a fundamental role in communication with political impact. We are talking about expressives, that is, expressions that are used to communicate a certain attitude. The purpose of this special issue of Daimon is to offer an overview of some of the debates that are currently taking place in relation to the political dimension of expressives, but also of related discussions that sometimes overlap with it, both in philosophy of language and in related branches of philosophy.

Keywords: expressives, philosophy of language, political turn in analytic philosophy, injustice
\end{abstract}

Recibido: 02/07/2021. Aceptado: 02/07/2021.

* Investigador postdoctoral, Departamento de Filosofía I, Universidad de Granada. Correo electrónico: edperez@ ugr.es.

** Catedrática de Lógica y Filosofía de la Ciencia, Departamento de Filosofía I, Universidad de Granada. Correo electrónico: frapolli@ugr.es. 
Desde sus orígenes, la filosofía analítica ha pretendido ofrecer un tratamiento universal de los rasgos característicos de fenómenos humanos como el conocimiento o el lenguaje. Por este motivo, ha dejado de lado las particularidades que surgen cuando estas capacidades se ponen en práctica en contextos no ideales y ha asumido, por ejemplo, que, a igualdad de evidencia y capacidades, todas las personas que participan en un intercambio epistémico tienen la misma credibilidad. Desde hace unos años, sin embargo, se ha producido un movimiento de acercamiento a las prácticas reales y de huida de las idealizaciones no justificadas que pretende poner las herramientas conceptuales desarrolladas durante el último siglo al servicio de la justicia social. Un paso dentro de este movimiento es la introducción en la epistemología contemporánea del concepto de injusticia epistémica (Fricker, 2007). La variedad de injusticia correspondiente en filosofía del lenguaje es la injusticia discursiva (Kukla, 2014) o injusticia del habla (Ayala, 2016), ambos conceptos herederos de las discusiones sobre los mecanismos lingüísticos detrás de la pornografía que tuvieron lugar en la década de los noventa (Langton, 1993; Hornsby y Langton, 1998).

En este ámbito, el giro político de la filosofía analítica ha pasado también por el análisis de expresiones del lenguaje natural que, por no encajar de forma completamente satisfactoria con la concepción del significado como condiciones de verdad, han recibido tradicionalmente poca atención desde la filosofía del lenguaje. Sin embargo, estas expresiones juegan un papel fundamental en la comunicación con impacto político. Hablamos de los expresivos. Llamamos "expresivos" a aquellas expresiones del lenguaje natural que se utilizan para comunicar una cierta actitud. Si lo que nos interesa de los expresivos es su potencial para el uso político, son especialmente relevantes expresiones como los slurs (véanse, por ejemplo, Potts, 2007; Hom, 2008; Camp, 2013; Jeshion, 2013; Cepollaro y Stojanovic, 2016), los dogwhistles (Saul, 2018) y los términos étnicos y sociales neutros usados como insultos o TESNI (véase Castroviejo, Fraser y Vicente, 2020), así como las insinuaciones (véanse Fricker, 2012; Camp, 2018). Los slurs son insultos que se dirigen específicamente a un determinado grupo social. Como tales, estas expresiones son sumamente dañinas, pero poco sutiles. Los dogwhistles, por el contrario, no son generalmente percibidos como herramientas que comuniquen una actitud, lo que los hace potencialmente más peligrosos. Por ejemplo, utilizar expresiones como "PER" puede movilizar ciertas actitudes contra andaluces y extremeños en el auditorio, pero de forma tal que al menos una parte del mismo ignorará que esto está ocurriendo. Los TESNI, por su parte, son términos en principio neutrales que se utilizan en ocasiones para transmitir estereotipos negativos. Las insinuaciones, finalmente, son un tipo particular de inferencia pragmática.

El propósito de este número especial de Daimon, resultado de una iniciativa de las personas participantes en el proyecto de investigación de excelencia de la Junta de Andalucía "La identificación inferencial de las proposiciones. Una reconsideración de las dicotomías clásicas en metafísica, semántica y pragmática” (P18-FR-2907), es ofrecer una panorámica de algunos debates que se están desarrollando en la actualidad en relación con la dimensión política de los expresivos, pero también de discusiones cercanas que en ocasiones se solapan con esta, tanto en filosofía del lenguaje como en ramas de la filosofía afines.

Los artículos incluidos en este número especial se han ordenado siguiendo una narrativa que parte de temáticas clásicas dentro de este tipo de discusiones para ir abriendo el espectro hacia debates menos estándar que superan finalmente los límites de la filosofía del lenguaje. 
El de Thaddeus Metz ("Exactly why are slurs wrong?") y el de Alba Moreno y Eduardo Pérez-Navarro ("The resistant effect of slurs: A nonpropositional, presuppositional account") se centran en viejos conocidos como son los slurs, si bien el primero deja de lado la discusión semántica para investigar las razones por las que ciertos usos de estas expresiones son moralmente incorrectos. Los siguientes artículos exploran fenómenos lingüísticos similares que solo recientemente se han empezado a describir, sean los TESNI, como en el de David Bordonaba-Plou y José Ramón Torices ("Paving the road to Hell: The Spanish word menas as a case study"), o las insinuaciones provocativas, como en el de Álvaro Domínguez-Armas y Andrés Soria-Ruiz ("Provocative insinuations"). Dentro de la filosofía del lenguaje encontramos también el artículo de Claudia Picazo ("Not all speakers are equal: Harm and conversational standing”) y el de Manuel Almagro y Neftalí Villanueva ("Exactly, what do you mean?"), que exploran la influencia que distintos aspectos del contexto tienen a la hora de determinar la fuerza con la que se implementa una norma de permisibilidad, en el primer caso, y de clasificar un uso del lenguaje como evaluativo, en el segundo. Finalmente, el artículo de María J. Frápolli y Llanos Navarro ("I am large, I contain multitudes: Epistemic pragmatism, testimonial injustice and positive intersectionalism”) permite a este número ampliar su foco para cubrir otra de las protagonistas del giro político en filosofía analítica: la epistemología. El número se completa con una reseña a cargo de Javier Moscoso de Excitable Speech: A Politics of the Performative, de Judith Butler, con motivo de su reedición en Routledge Classics. En lo que sigue, recorremos con un poco más de detalle los contenidos del número.

Como se ha adelantado anteriormente, la pregunta que guía el artículo de Thaddeus Metz es por qué es moralmente incorrecto usar un slur en los casos en los que lo es. Metz considera tres posibles explicaciones: la bienestarista, la kantiana y una teoría relacional que toma prestadas ideas de la filosofía sudafricana. Es esta última opción la que se va a defender como más adecuada frente a las desventajas que presentan las otras dos alternativas. El artículo de Alba Moreno y Eduardo Pérez-Navarro, por su parte, tiene un propósito doble. Por un lado, se propone describir de manera unificada el comportamiento que hace de los slurs expresiones particulares. La peculiaridad se achaca, en concreto, a su carácter resistente frente a la cancelación, el rechazo y la retractación. Por otro lado, el artículo pretende explicar este comportamiento a través de una teoría presuposicional del significado de los slurs que, como otras propuestas recientes, entiende el contenido presupuesto en estos casos como no proposicional.

Si los dos primeros artículos de este número se centran en los slurs, los dos siguientes se ocupan de fenómenos relacionados pero mucho menos discutidos. Por un lado, David Bordonaba-Plou y José Ramón Torices argumentan a partir de resultados experimentales que la palabra "mena" exhibe los rasgos característicos de los TESNI y exploran las distintas direcciones en las que el uso de la palabra podría evolucionar. En algunos de estos casos la palabra seguiría siendo un TESNI, mientras que en otros se convertiría en un slur. Por otro lado, Andrés Soria-Ruiz y Álvaro Domínguez-Armas tratan de localizar el mecanismo que se halla detrás de ciertas inferencias racistas. Tras descartar que se trate de slurs, TESNI, dogwhistles o implicaturas conversacionales, sugieren explicar estas inferencias como insinuaciones provocativas.

El artículo de Claudia Picazo y el de Manuel Almagro y Neftalí Villanueva se encuadran también dentro de la filosofía del lenguaje, en esta ocasión con el contexto como protago- 
nista. En su artículo, Claudia Picazo argumenta que la fuerza de las normas que determinan qué es permisible en un determinado contexto es sensible a la posición conversacional del hablante y que, en casos extremos, esta posición puede ser insuficiente para establecer ningún tipo de norma. Por su parte, el artículo de Manuel Almagro y Neftalí Villanueva pretende, por un lado, rastrear la relación que la literatura atribuye al contexto y los usos evaluativos del lenguaje y, por otro, argumentar que algunas de las propuestas teóricas que actualmente se encuentran en el mercado son incapaces de explicar los hallazgos experimentales recientes.

El artículo de María J. Frápolli y Llanos Navarro, por último, contrapone dos concepciones del conocimiento: el pragmatismo epistémico de Brandom y la concepción del conocimiento implícita en la obra de Fricker y quienes han seguido su estela. Frápolli y Navarro argumentan que, por sí solas, estas dos concepciones del conocimiento están en tensión, pero que pueden hacerse compatibles si se complementan con un "interseccionalismo positivo".

Los artículos contenidos en este número especial parten de debates de gran actualidad y contribuyen a ellos con propuestas novedosas que exploran los límites del aparataje teórico disponible hasta el momento. Sea poniendo sobre la mesa preguntas hasta ahora ignoradas sobre fenómenos ampliamente explotados (¿por qué son moralmente incorrectos algunos usos de los slurs?), contribuyendo a la incipiente literatura sobre fenómenos de reciente descripción, como los TESNI o las insinuaciones provocativas, aprovechando los avances de la filosofía experimental o poniendo en diálogo tradiciones que se han dado la espalda durante largo tiempo, como el pragmatismo epistémico de Brandom y la ética del conocimiento, estos artículos suponen un paso adelante en la aplicación de la filosofía contemporánea a problemas que deberían preocupar a cualquier persona interesada en cuestiones sociales y políticas. Esperamos, por tanto, que estos trabajos sean la base sobre la que puedan proponerse herramientas de intervención en los ámbitos abarcados por el número.

\section{Referencias}

Ayala, S. (2016), "Speech affordances: A structural take on how much we can do with our words", European Journal of Philosophy, 24(4), 879-891.

Camp, E. (2013), "Slurring perspectives”, Analytic Philosophy, 54(3), 330-349.

Camp, E. (2018), "Insinuation, common ground, and the conversational record", en D. Fogal, D. W. Harris \& M. Moss (eds.), New Work on Speech Acts, Oxford: Oxford University Press, pp. 40-66.

Castroviejo, E., Fraser, K. \& Vicente, A. (2020), “More on pejorative language: Insults that go beyond their extension", Synthese.

Cepollaro, B. \& Stojanovic, I. (2016), "Hybrid evaluatives: In defense of a presuppositional account", Grazer Philosophische Studien, 93(3), 458-488.

Fricker, E. (2012), "Stating and insinuating", Proceedings of the Aristotelian Society Supplementary Volume, 86(1), 61-94.

Fricker, M. (2007), Epistemic Injustice: Power and the Ethics of Knowing, Oxford: Oxford University Press.

Hom, C. (2008), "The semantics of racial epithets", The Journal of Philosophy, 105(8), 416-440. 
Jeshion, R. (2013), "Slurs and stereotypes", Analytic Philosophy, 54(3), 314-329.

Kukla, R. (2014), "Performative force, convention, and discursive injustice", Hypatia, 29(2), 440-457.

Langton, R. (1993), "Speech acts and unspeakable acts", Philosophy and Public Affairs, 22(4), 293-330.

Hornsby, J. \& Langton, R. (1998), "Free speech and illocution”, Legal Theory, 4(1), 21-37.

Potts, M. (2007), “The expressive dimension”, Theoretical Linguistics, 33(2), 165-198.

Saul, J. (2018), "Dogwhistles, political manipulation, and philosophy of language", en D. Fogal, D. W. Harris \& M. Moss (eds.), New Work on Speech Acts, Oxford: Oxford University Press, pp. 360-384. 